\title{
Case Report \\ Prebiliary Right Hepatic Artery Resulting in Common Hepatic Duct Compression and Subsequent Intrahepatic Stone Formation: Myth or Reality?
}

\author{
Vanessa Marron Mendes, ${ }^{1}$ Haydar A. Nasser, ${ }^{1}$ Georges Bou Nassif, ${ }^{2}$ and Ali Choukr ${ }^{2}$ \\ ${ }^{1}$ Université Libre de Bruxelles (ULB), 1000 Brussels, Belgium \\ ${ }^{2}$ Lebanese University, Beirut 961, lebanon \\ Correspondence should be addressed to Haydar A. Nasser; derdyail@hotmail.com
}

Received 10 October 2013; Revised 11 December 2013; Accepted 11 December 2013; Published 9 January 2014

Academic Editor: Gianfranco D. Alpini

Copyright ( 2014 Vanessa Marron Mendes et al. This is an open access article distributed under the Creative Commons Attribution License, which permits unrestricted use, distribution, and reproduction in any medium, provided the original work is properly cited.

\begin{abstract}
The vascular anatomy of the liver is subjected to many variations. Aberrant hepatic artery is not an uncommon finding during visceral surgery; however, topographic variations are less reported in the literature. Prebiliary artery crossing anteriorly to the common hepatic duct was firstly reported in 1984. We present here a case of a 52-year-old lady who presented with obstructive jaundice and right upper quadrant pain. Paraclinical investigations were consistent with intrahepatic stones and a benign stricture on the CBD. During surgery, a prebiliary right hepatic artery compressing the CHD was noted. The liver pedicle was dissected and a hepaticojejunostomy was performed that resulted in a good outcome after 24 months of followup.
\end{abstract}

\section{Introduction}

In the 20th century, Michels [1] and Hiatt et al. [2] proposed internationally recognized schemes for the classification of the hepatic arterial anatomy with its variations. In a retrospective study, 604 selective angiographies showed that the conventional vascular anatomy of the liver was found in $79.1 \%$ of individuals [3]. Normally, the hepatic artery courses between the extrahepatic bile duct and the portal vein. Aberrant and replaced right hepatic arteries (RHA) are wellknown variants. Topographic variations, such as anterior or retroportal RHA, have been rarely described before [47]. The first case of Jaundice, and hepatolithiasis due to an anterior RHA pressing on the CBD was reported in 1984. Since then, one similar case was reported [8] and the current one is to be added.

\section{Case Report}

A 52-year-old lady was referred to general surgery clinics because of 2 months history of jaundice and right upper quadrant pain. She has no previous medical history. Clinically the patient was stable hemodynamically, without any signs of sepsis. On CT-scan, the bile ducts were dilated and filled with stones. The MRCP was not of diagnostic value due to motion artifacts. The total serum bilirubin increased to $5 \mathrm{mg} / \mathrm{dL}$ from a baseline of $2.9 \mathrm{mg} / \mathrm{dL}$. She was offered ERCP twice without diagnostic yield, and her symptoms persisted despite sphincterotomy. Alkaline phosphatase serum level was also elevated ( 3 times of normal). The decision was to proceed with an exploratory laparotomy in order to relieve the symptoms and establish a diagnosis. This treatment option was discussed with the patient, and she had signed the informed consent the night before the procedure.

At laparotomy, an aberrant anterior right hepatic artery pressing on the CHD was noted. The CHD was dilated just proximal to the crossing point (Figures 1(a) and 1(b)). The artery was dissected off the CHD. An intraoperative cholangiography showed intrahepatic ducts filled with stones (Figure 2). We performed a choledochotomy to facilitate the complete evacuation of these dark-brown stones (Figure 3). A hepaticojejunostomy was then fashioned. The post-operative 


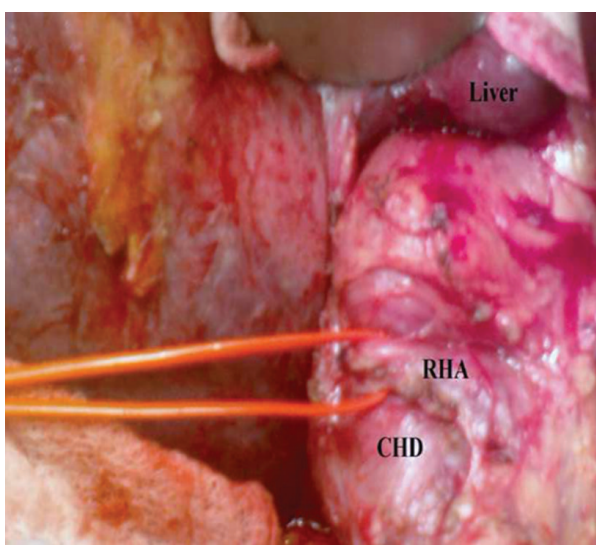

(a)

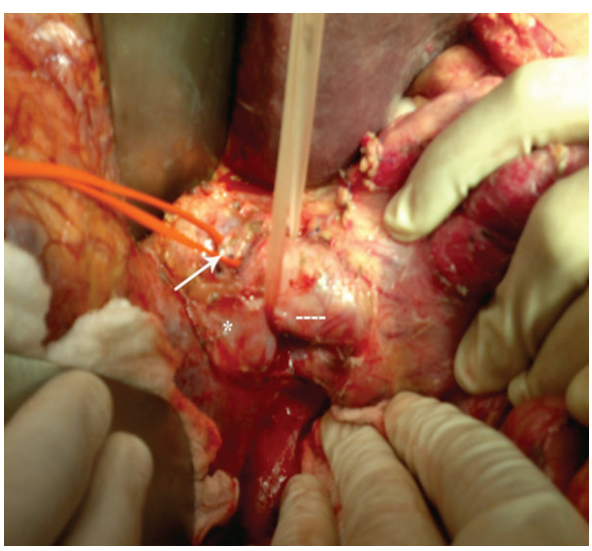

(b)

FiguRE 1: (a) RHA (red lacks) crossing anteriorly to the CHD and compressing it. RHA: right hepatic artery; CHD: hepatic duct. (b) Lateral view of the hepaticoduodenal ligament. The RHA (white arrow) crossing anteriorly to the dilated CHD (dashed line). Asterisk corresponds to the dilated right hepatic duct.

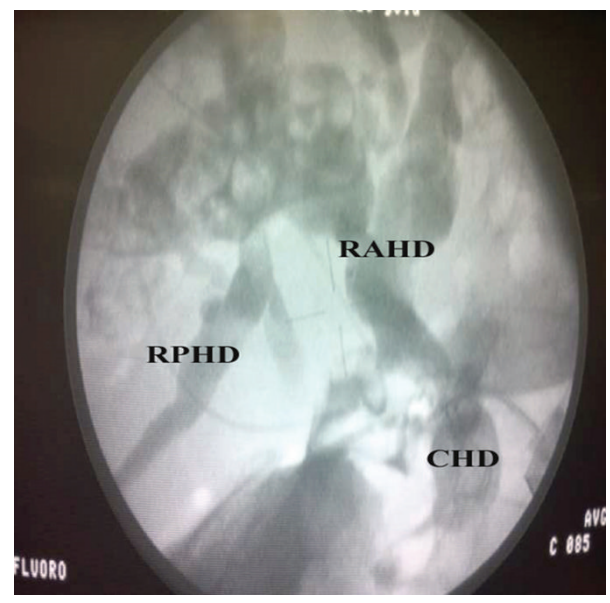

FIGURE 2: Intraoperative cholangiogram showing the dilated common hepatic duct (CHD), right posterior hepatic duct (RPHD), and the right anterior hepatic duct (RAHD).

course was uneventful and the patient has had her symptoms completely resolved within 2 weeks. We are now at 24 months after surgery with no signs of recurrence.

\section{Discussion}

Pseudoobstruction of the CHD by a pulsatile RHA has been previously documented on MRCP and should not be misdiagnosed as obstructing periampullary tumors [10]. Usually, the absence of obstructive symptoms confirms this diagnosis. However, jaundice due to pulsatile RHA has been reported previously $[5,6,8,9,11]$, and this is known as the right hepatic artery syndrome [5]. The RHA usually runs posteriorly to the bile duct and can result in an inflammatory impingement of the CHD $[5,6,10]$. Two authors $[8,9]$ have described 3 cases with this syndrome but with distinguishing features: anterior RHA and hepatolithiasis. Tsuchiya et al. [9]

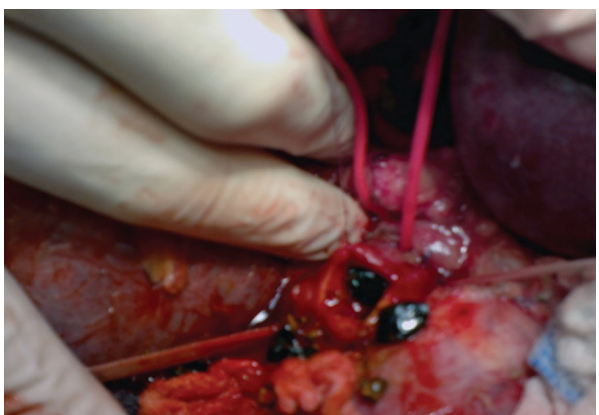

FIGURE 3: Choledochotomy and evacuation of the pigmented stones.

and Baek et al. [8] attributed jaundice and gallstone formation to an aberrant RHA running anteriorly to the CBD. Whether or not compression of the CHD has led to stones formation is questionable. The first case report was criticized by Professor G.B. Ong [9]; he stated that intrahepatic stones formation preceded the obstructive symptoms and that the stones got impacted within the CHD, at the level of the arterial crossing. However, if pulsatile compression is an acceptable theory (the right hepatic artery syndrome), then subsequent intrahepatic stone formation should also be regarded as an acceptable one; partial or complete obstruction of the $\mathrm{CBD} / \mathrm{CHD}$ leads to bile stasis and later on to bacterial proliferation [12]. One experimental study showed that liver bacterial concentration was significantly elevated after bile duct ligation [13]. Bacteria can reach the liver either by translocation from the gut or through the blood [12] and result in the so called pigmented or dark-brown gallstones formation [14]; as a result of this sequence analysis, compression of the CHD by an aberrant RHA could lead to bile stasis ande then bacterial proliferation and ends with gallstone formation. In 3 reported cases of right hepatic artery syndrome $[5,6,11]$, the RHA was running posteriorly to the $\mathrm{CBD}$ and none of the authors described intrahepatic gallstones. Another important feature in Tsuchiya et al., report [9] that is worth mention was 
TABle 1: Different treatment modalities adopted in patients with hepatolithiasis due to right hepatic artery syndrome.

\begin{tabular}{lcccc}
\hline Author [reference] & $n$ & Operations & Stones aspect & Postoperative course \\
\hline Baek et al. [8] & 1 & Hepaticojejunostomy & N/A & Uneventful \\
\hline Tsuchiya et al. [9] & 2 & $\begin{array}{c}\text { Hepaticojejunostomy in } \\
\text { case 1 }\end{array}$ & $\begin{array}{c}\text { Peft partial hepatectomy } \\
\text { + hepatico-jejunostomy } \\
\text { in case 2 }\end{array}$ & $\begin{array}{c}\text { Pigmented } \\
\text { Case 2: patient died on day 53 after }\end{array}$ \\
\hline
\end{tabular}

(n): number of patients; N/A: not available.

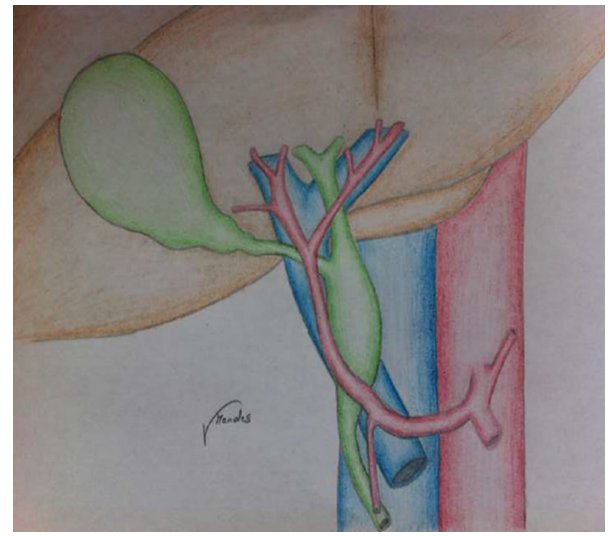

FIGURE 4: Schematic diagram illustrating the compression on the CBD exerted by the anterior aberrant RHA.

the absence of gallstones distal to the compression site. This was also an intraoperative finding cited by Baek and colleagues [8]. Table 1 summarizes the treatment modalities adopted in each case. In our case, choledochotomy followed with Roux-en-Y hepaticojejunostomy resulted in a good outcome. To the best of our knowledge, this is the 4th case in the literature where an anterior crossing RHA results in biliary stasis and hepatolithiasis. The dark-brown aspect of the stones observed in our case and elsewhere [9] is highly suggestive of their infectious nature. This was probably due to the cord-like effect that was exerted by the aberrant RHA on the CBD (Figure 4).

\section{Conflict of Interests}

The authors declare that there is no conflict of interests regarding the publication of this paper.

\section{References}

[1] N. A. Michels, "Newer anatomy of the liver and its variant blood supply and collateral circulation," The American Journal of Surgery, vol. 112, no. 3, pp. 337-347, 1966.

[2] J. R. Hiatt, J. Gabbay, and R. W. Busuttil, "Surgical anatomy of the hepatic arteries in 1000 cases," Annals of Surgery, vol. 220, no. 1, pp. 50-52, 1994.

[3] A. Koops, B. Wojciechowski, D. C. Broering, G. Adam, and G. Krupski-Berdien, "Anatomic variations of the hepatic arteries in 604 selective celiac and superior mesenteric angiographies,"
Surgical and Radiologic Anatomy, vol. 26, no. 3, pp. 239-244, 2004.

[4] S. Honma, W. Matsuda, and M. Kudo, "Right hepatic artery traveling anteriorly to the common bile duct," Anatomical Science International, vol. 88, pp. 93-96, 2013.

[5] K. Miyashita, K. Shiraki, T. Ito, H. Taoka, and T. Nakano, "The right hepatic artery syndrome," World Journal of Gastroenterology, vol. 11, no. 19, pp. 3008-3009, 2005.

[6] D. Eshtiaghpour, V. E. Eysselein, and S. Reicher, "Hepatic artery compression of the common hepatic duct: direct visualization by single-operator peroral cholangioscopy," Clinical Gastroenterology and Hepatology, vol. 10, no. 5, article e45, 2012.

[7] S. Wadhwa, G. Khorwal, and S. Rachel Tigga, "Retroportal proper hepatic artery with malrotated gut," Anatomical Science International, vol. 88, no. 4, pp. 242-245, 2013.

[8] Y. H. Baek, S. R. Choi, J. H. Lee et al., "Obstructive jaundice due to compression of the common bile duct by right hepatic artery originated from gastroduodenal artery," The Korean Journal of Gastroenterology, vol. 52, no. 6, pp. 394-398, 2008.

[9] R. Tsuchiya, T. Eto, and N. Harada, "Compression of the common hepatic duct by the right hepatic artery in intrahepatic gallstones," World Journal of Surgery, vol. 8, no. 3, pp. 321-326, 1984.

[10] Y. Watanabe, M. Dohke, T. Ishimori et al., "Pseudo-obstruction of the extrahepatic bile duct due to artifact from arterial pulsatile compression: a diagnostic pitfall of MR cholangiopancreatography," Radiology, vol. 214, no. 3, pp. 856-860, 2000.

[11] K. Izuishi, Y. Toyama, H. Wakabayashi, H. Usuki, and H. Maeta, "Compression of the common hepatic duct by the right hepatic artery," Clinical Imaging, vol. 29, no. 5, pp. 342-344, 2005.

[12] H. G. Beger and A. Schwarz, "Spectrum of biliary infections in the West and in the East," HPB Surgery, vol. 8, pp. 215-222, 1995.

[13] J.-H. Chuang, S.-Y. Lee, W.-J. Chen, C.-S. Hsieh, N.-K. Chang, and S.-K. Lo, "Changes in bacterial concentration in the liver correlate with that in the hepaticojejunostomy after bile duct reconstruction: implication in the pathogenesis of postoperative cholangitis," World Journal of Surgery, vol. 25, no. 12, pp. 1512-1518, 2001.

[14] L. A. Wetter, R. M. Hamadeh, J. M. Griffiss, A. Oesterle, B. Aagaard, and L. W. Way, "Differences in outer membrane characteristics between gallstone-associated bacteria and normal bacterial flora," The Lancet, vol. 343, no. 8895, pp. 444-448, 1994. 


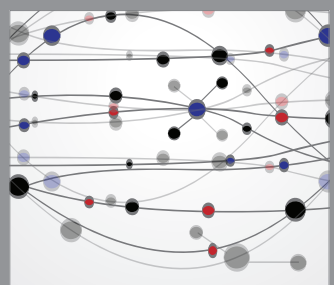

The Scientific World Journal
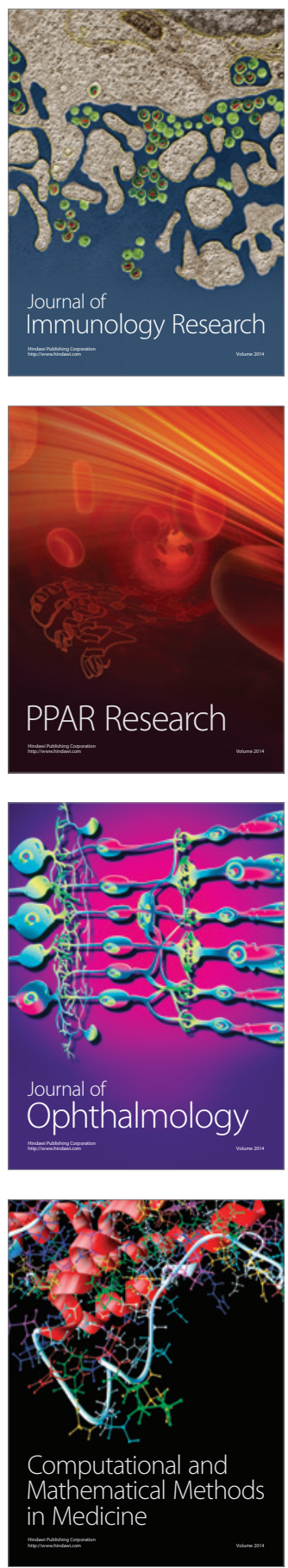

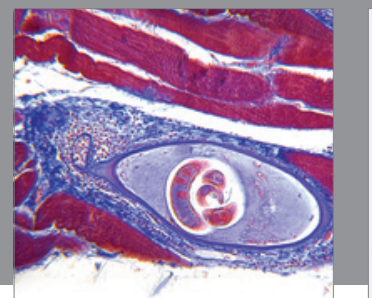

Gastroenterology

Research and Practice
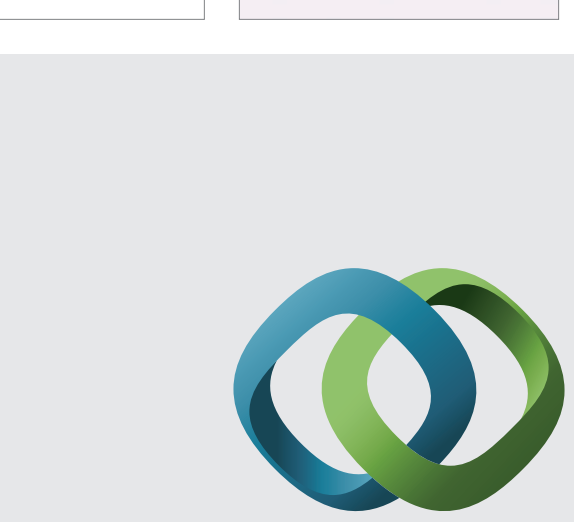

\section{Hindawi}

Submit your manuscripts at

http://www.hindawi.com
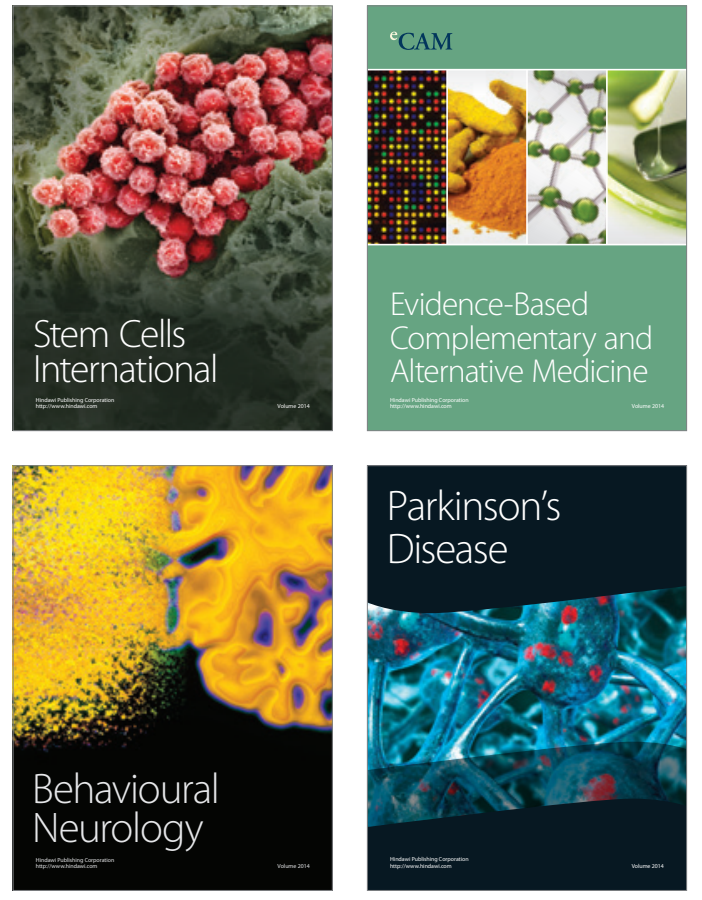
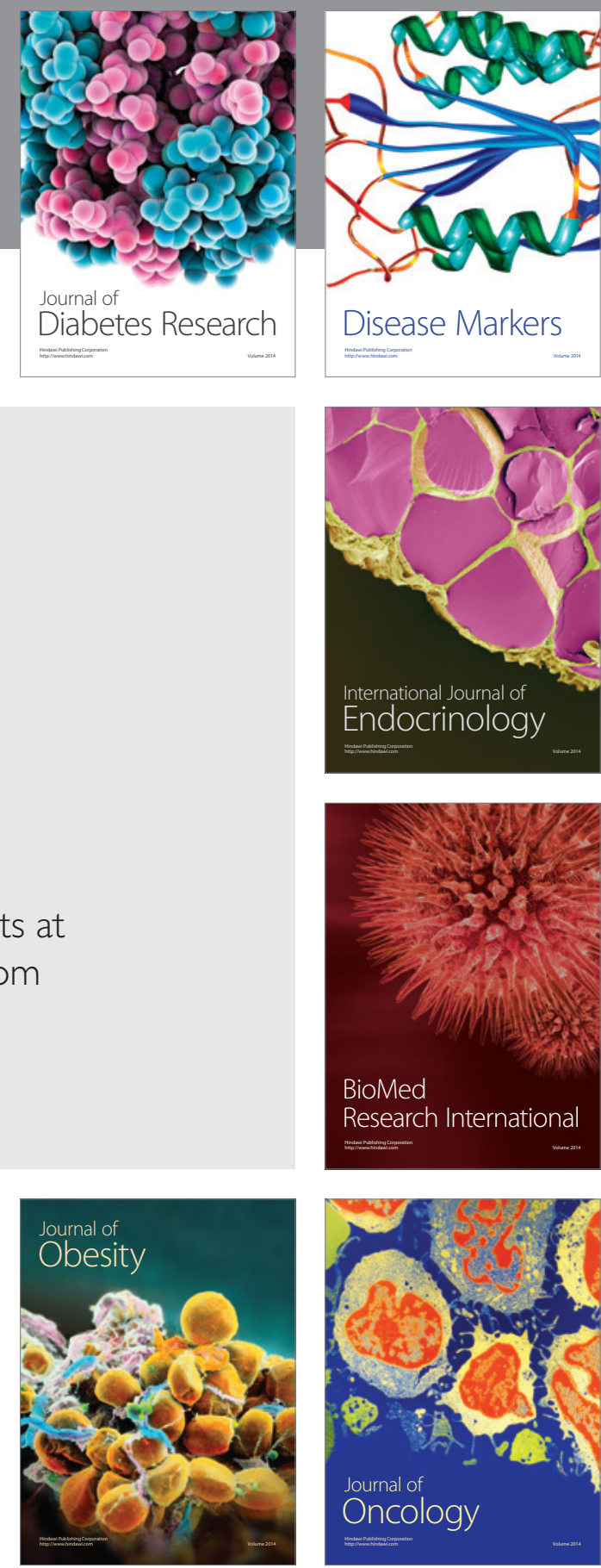

Disease Markers
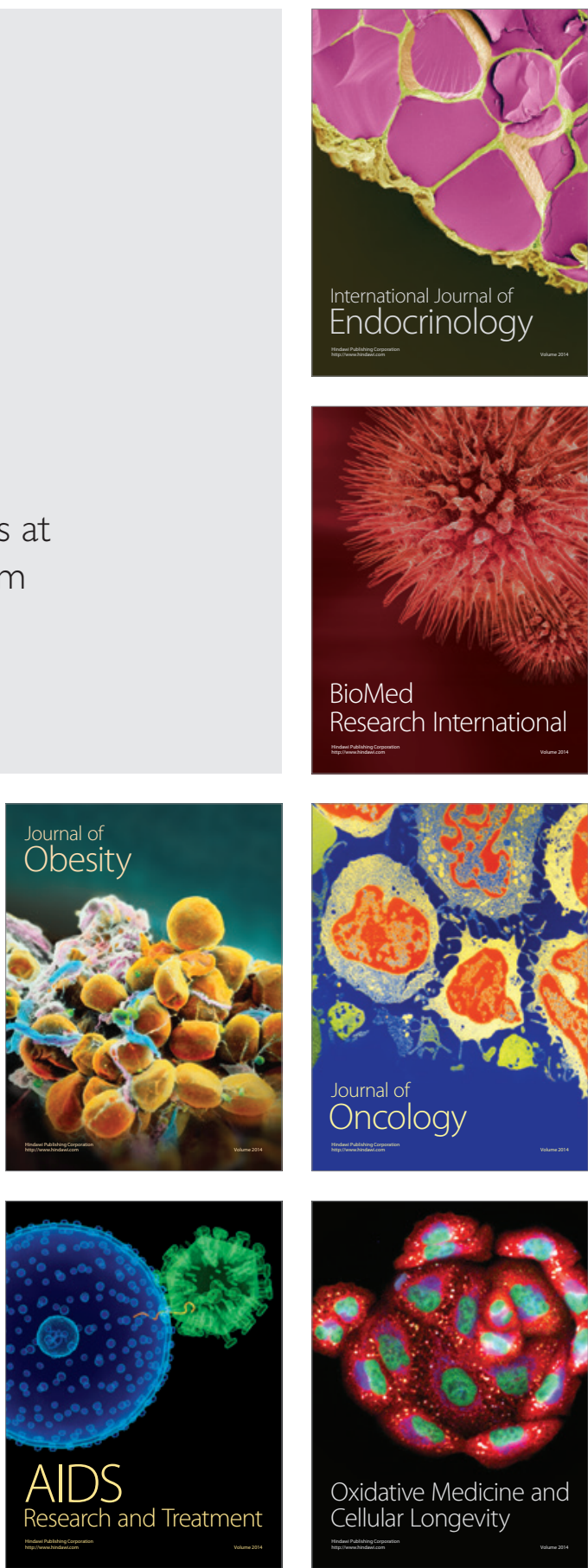